\title{
A GENERALIZATION OF A THEOREM OF S. PICCARD
}

\author{
WOLFGANG SANDER
}

The following theorem is due to S. Piccard [2, p. 30]:

"The difference of two second category Baire sets (see [1]) contains a nonempty open set".

For various generalizations of this result the reader is referred to [3] and [4], where he can also find some more references.

In this note we give a short proof of a generalization of Piccard's theorem.

Let $f: X \times X \rightarrow X$. We define $f_{x}: X \rightarrow X$ and $f^{y}: X \rightarrow X$ by $f_{x}(y)=f(x, y)$ and $f^{y}(x)=f(x, y)$ for all $x, y \in X$. Then $f$ is globally solvable, if $f$ is continuous and if there exist two continuous functions $\psi, \phi: X \times X \rightarrow X$ such that $f(x, y)=z$ is equivalent to $x=\psi(y, z)$ and $y=\phi(x, z)$ for all $x, y$, $z \in X$. It follows that $f_{x}, f^{y}, \psi^{z}, \phi^{z}$ are homeomorphisms.

If $X$ is a topological group and $f(x, y)=x \cdot y$, then $\psi(y, z)$ and $\phi(x, z)$ may be taken to be $z \cdot y^{-1}$ and $x^{-1} \cdot z$.

THEOREM (CF. [4, SATZ 7]). Let $X$ be a topological space and let $f: X \times X \rightarrow$ $X$ be a globally solvable function. If $A, B \subset X$ are of second category and $A$ has the property of Baire, then $f(A \times B)$ contains a nonempty open set and $X$ is a Baire space.

Proof. By hypothesis there exists a nonempty open set $G$ such that $G-A$ is of first category. For any set $C \subset X$ let $D(C)$ denote the set of all points of $X$ where $C$ is of second category. Then $D(C) \neq \varnothing$ if and only if $C$ is of second category, $G \cap D(C)=G \cap D(G \cap C)$ and $D(G \cap C)=D(G \cap A$ $\cap C)[1$, pp. 83-85]. Thus

$$
f(G \times D(B))=\bigcup\left\{f^{y} G: y \in D(B)\right\}
$$

is a nonempty open set. Since $D \gamma=\gamma D$ for each homeomorphism $\gamma$ of $X$, $\varnothing \neq f(G \times D(B))=\left\{z \in X: G \cap \psi^{z} D(B) \neq \varnothing\right\}=\left\{z \in X: G \cap D\left(\psi^{z} G\right)\right.$ $\neq \varnothing\}=\left\{z \in X: G \cap D\left(G \cap \psi^{2} B\right) \neq \varnothing\right\}=\{z \in X: G \cap D(G \cap A \cap$ $\left.\left.\psi^{z} B\right) \neq \varnothing\right\} \subset\left\{z \in X: A \cap \psi^{z} B \neq \varnothing\right\}=f(A \times B)$.

The global solvability of $f$ implies that $X$ is a homogeneous space. Since $D(X)$ is invariant under every homeomorphism of $X$, it follows that $D(X)=$ $X$, that is, $X$ is a Baire space.

Received by the editors May 10, 1978 and, in revised form, June 30, 1978.

AMS (MOS) subject classifications (1970). Primary 22A10.

Key words and phrases. Baire space, Baire set, second category set.

(c) 1979 American Mathematical Society 0002-9939/79/0000-0078/\$01.50 
ACKNOWLEDGEMENT. The author wishes to thank the referee for comments and advice.

\section{REFERENCES}

1. K. Kuratowski, Topology. Vol. 1, Academic Press, New York; PWN, Warszawa, 1966.

2. S. Piccard, Sur les ensembles de distances des ensembles de points d'un expace euclidien, Mém. Univ. Neuchâtel, Vol. 13, Secrétariat de l'Université Neuchâtel, 1939.

3. K. P. S. Bhaskara Rao and M. Bhaskara Rao, On the difference of two second category Baire sets in a topological group, Proc. Amer. Math. Soc. 47 (1975), 257-258. MR 50 \#2386.

4. W. Sander, Verallgemeinerungen eines Satzes von S. Piccard, Manuscripta Math. 16 (1975), 11-25. MR 51 \#9018.

Institut C pür Mathematik, Technische Universttät Braunschweig, D 33 BraunschWEig, DeutschLAND 